\title{
Adaptive Control of a Seeker-Guided 2D Missile with Unmodeled Aerodynamics
}

\author{
Anthony M. D'Amato, and Dennis S. Bernstein ${ }^{\dagger}$ \\ University of Michigan, 1320 Beal Ave., Ann Arbor, MI 48109
}

\begin{abstract}
In this paper we apply extensions of retrospective cost adaptive control (RCAC) to a $2 \mathrm{D}$ missile model considered in prior papers as a benchmark test of adaptive control methods. The dynamics of the missile are highly nonlinear, and instantaneous linearizations are nonminimum phase due to nose sensing and tail actuation. The results that we present in this paper show that the RCAC controller provides results that are comparable to a highly tuned autopilot based on aerodynamic modeling, whereas the RCAC controller uses no knowledge of the missile's aerodynamics. These results significantly improve the results obtained on the same problem using an earlier version of RCAC, presented at the 2010 GNC.
\end{abstract}

\section{Nomenclature}

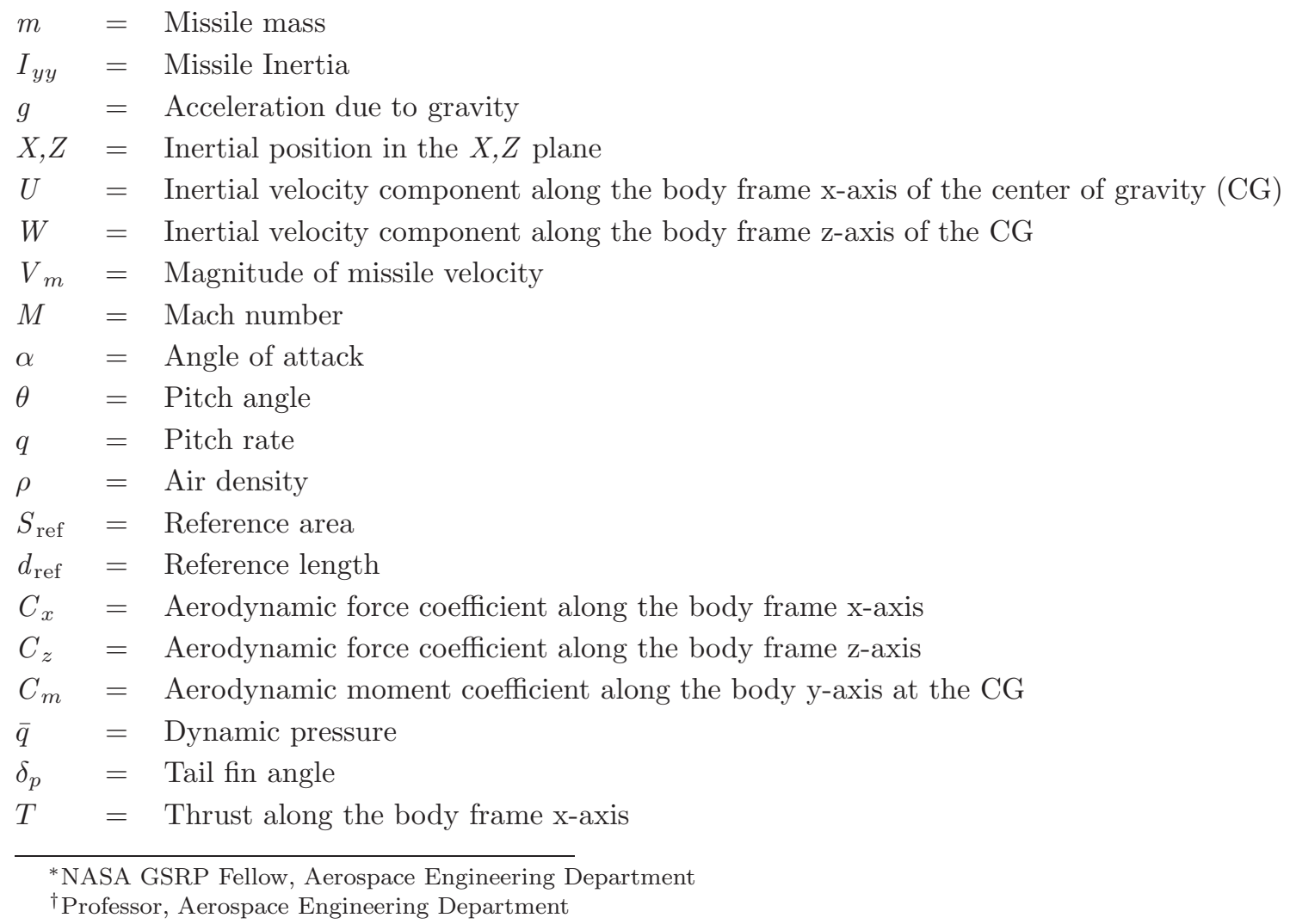




\section{Introduction}

Many difficulties are encountered when applying adaptive control to a tail-controlled missile system. One of the issues encountered is nonminimum-phase zeros in the linearizations about trim setpoints; these zeros are are due to tail actuation and sensor suites located ahead of the center of gravity. Nonminimumphase zeros might also appear in the digitized system once the continuous-time plant is sampled during flight. Therefore, standard techniques for minimum-phase systems $[1,2]$ are not well-suited for missile control. Additionally, actuator delays, aerodynamic effects, and structural flexibility introduce significant complexity to the problem. Furthermore, as described in [3], tail-controlled missiles require fast response times in order to achieve acceleration commands generated by guidance control laws.

In previous work [3], cumulative retrospective cost adaptive control (RCAC) [4,5], was implemented on a 2D missile as an outer-loop with the three-loop-autopilot, found in [6], as the inner loop. The cumulative retrospective cost adaptive control algorithm requires knowledge of nonminimum-phase zero locations. Since the linearized missile dynamics in closed loop with the three-loop autopilot has a nonminimum-phase zero, this information was scheduled in order to implement the adaptive controller.

The present work differs from the work in [3] in several ways. First, the inner-loop is eliminated, specifically, the missile is controlled only by the retrospective cost adaptive control (RCAC) algorithm. Secondly, we do not schedule the nonminimum-phase zero location. Recent extensions to the RCAC algorithm eliminate the requirement for knowledge of the nonminumum-phase zero locations, as described in $[7,8]$, specifically, the algorithm development requires a limited number of Markov parameters. Furthermore, in the SISO case, the algorithm has been shown to be robust to the magnitude of the Markov parameter, meaning that sign information usually suffices. Therefore, we do not schedule the estimate of the required Markov parameter; a single value is used for all trim conditions. It should be noted that this parameter does not depend on any aerodynamic modeling information specific to the 2D missile. Details about the required modeling information are discussed in [8]. Furthermore, to reduce oscillation of the missile body during the flight, we include rate saturation in the retrospective cost function.

In the present work, we design a set of scenarios and compare the performance of the retrospective cost adaptive controller to the three-loop-autopilot performance. We examine the nominal scenario, in which, the autopilot has exact knowledge of the missile's aerodynamic coefficients and dynamics. The adaptive controller tuning parameters are tuned to yield the best performance under the nominal scenario. Next, we perturb the aerodynamic coefficients using an affine linear transformation (shift and scale factor). In these scenarios, the autopilot remains tuned for the nominal scenario; the adaptive controller tuning parameters are also unchanged. Finally, we examine the effect of noisy sensors on the performance of both controllers, specifically, noise is added to the pitch rate measurement. In each scenario presented, a Monte Carlo simulation is used to determine the median miss distance, where the variation in simulations is due to the changing initial conditions of the target.

This paper is organized as follows. In Section II, we give a general problem formulation and define the tactical objective. In Section III, we present the nonlinear missile dynamics and actuator dynamics. In Section IV, a brief construction of the RCAC algorithm applied to the $2 \mathrm{D}$ missile problem is outlined. The generalized algorithm setup is given in Appendix A. In Sections V to Section VIII, we present the simulation setups and results of the Monte Carlo simulations. Finally, in Section IX we provide conclusions.

\section{Problem Formulation}

Consider the target dynamics

$$
\begin{aligned}
\dot{v}(t) & =f_{t}(v(t)), \\
v_{p}(t) & =\left[\begin{array}{ll}
I_{2} & 0_{2 \times 2}
\end{array}\right] v(t),
\end{aligned}
$$


where $v(t) \in \mathbb{R}^{4}$ is the inertial position and velocity of the target at time $t$, and $v_{p}(t) \in \mathbb{R}^{2}$ is the inertial position of the target. The boundary conditions are

$$
\begin{array}{r}
v(0) \neq 0, \\
v\left(T_{t}\right) \neq 0,
\end{array}
$$

where $T_{t}$ is the final time of the target flight.

Next consider the missile dynamics

$$
\begin{aligned}
\dot{x}(t) & =f_{m}(x(t))+g(u(t)), \\
x_{p}(t) & =\left[\begin{array}{ll}
I_{2} & 0_{2 \times 2}
\end{array}\right] x(t),
\end{aligned}
$$

where $x(t) \in \mathbb{R}^{4}$ is the inertial position and velocity of the missile at time $t$ and $x_{p}(t) \in \mathbb{R}^{2}$ is the inertial position of the missile. The goal is to determine the control $u(t)$, that minimizes $\left|v_{p}(t)-x_{p}(t)\right|$ at some time $t \leq T_{t}$.

\section{Nonlinear Missile Model}

Consider the nonlinear three-degree-of-freedom model

$$
\begin{aligned}
\dot{X} & =U \cos \theta+W \sin \theta, \\
\dot{Z} & =-U \sin \theta+W \cos \theta, \\
\dot{\theta} & =q, \\
\dot{U} & =\frac{1}{m} T+\frac{\bar{q} S_{\mathrm{ref}}}{m} C_{x}(\alpha, M)-q W-g \sin \theta, \\
\dot{W} & =\frac{\bar{q} S_{\text {ref }}}{m} C_{z}\left(\alpha, M, \delta_{p}\right)+q U+g \cos \theta, \\
\dot{q} & =\frac{\bar{q} S_{\text {ref }} d_{\text {ref }}}{I_{y y}} C_{m}\left(\alpha, M, \delta_{p}, q\right),
\end{aligned}
$$

where the dynamic pressure is given by $\bar{q}=\frac{1}{2} \rho V_{m}^{2}$ and angle of attack is defined as $\alpha=\operatorname{atan}(W / U)$. Measurements of the pitch rate $q$ and lateral missile acceleration are assumed to take place at the inertial measurement unit (IMU) location. The lateral acceleration at the IMU is related to that measured at the center of gravity through the equation

$$
A_{z, \mathrm{IMU}}=A_{z, \mathrm{CG}}-\dot{q} x_{\mathrm{IMU}},
$$

where $x_{\mathrm{IMU}}$ is the distance from the CG to IMU. Here we assume that the missile IMU is forward of the CG location. The nonlinear model in equations (5)-(11) is linearized about trim points in the region of operation for the purpose of autopilot controller design. Additionally, the second-order fin actuator model

$$
\delta_{p}(s)=\frac{\omega_{a}^{2}}{s^{2}+2 \lambda_{a} \omega_{a} s+\omega_{a}^{2}} u(s),
$$

is used, where $u(s)$ is the fin actuator command, $\omega_{a}$ and $\lambda_{a}$ represent the natural frequency and damping ratio, respectively, of the actuator dynamics.

\section{III.A. Three-Loop Autopilot}

We compare the adaptive controller with the three-loop auto-pilot [6], which is given by

$$
u(s)=K_{q} q(s)+\frac{1}{s}\left(K_{\theta} q(s)+K_{I}\left[K_{s s} A_{z, \mathrm{cmd}}-A_{z, \mathrm{IMU}}\right]\right),
$$


where $K_{q}, K_{\theta}, K_{I}, K_{s s}$ are control gains, and $A_{z, \mathrm{cmd}}$ is the lateral acceleration command for the IMU location provided by the seeker and the missile's guidance system. A proportional navigation guidance law generates the acceleration. The control gains $K_{q}, K_{\theta}, K_{I}$, and $K_{s s}$ are determined by modeling and analysis, and scheduled based on angle of attack and Mach number, therefore the autopilot is actually many separate controls, which are switched depending on trim condition. Note that in practice this continuous-time controller is digitized using sample and hold operations.

\section{Overview of Application of Retrospective Cost to the 2D Missile Problem}

In this Section we given a brief overview of the retrospective cost adaptive controller for the 2D

nonlinear missile model. Note that RCAC does not require digitization since, it is developed in discrete time.

\section{IV.A. Controller Construction}

The control $u(k)$ is given by the strictly proper time-series controller of order $n_{\mathrm{c}}$ given by

$$
u(k)=\sum_{i=1}^{n_{\mathrm{c}}} M_{i}(k) u(k-i)+\sum_{i=1}^{n_{\mathrm{c}}} N_{i}(k) y(k-i),
$$

where, for all $i=1, \ldots, n_{\mathrm{c}}, M_{i}(k) \in \mathbb{R}^{l_{u} \times l_{u}}$ and $N_{i}(k) \in \mathbb{R}^{l_{u} \times l_{y}}$. The control (16) can be expressed as

$$
u(k)=\theta(k) \phi(k-1),
$$

where

$$
\theta(k) \triangleq\left[M_{1}(k) \cdots M_{n_{\mathrm{c}}}(k) N_{1}(k) \cdots N_{n_{\mathrm{c}}}(k)\right] \in \mathbb{R}^{l_{u} \times n_{\mathrm{c}}\left(l_{u}+l_{y}\right)}
$$

and

$$
\phi(k-1) \triangleq\left[\begin{array}{c}
u(k-1) \\
\vdots \\
u\left(k-n_{\mathrm{c}}\right) \\
y(k-1) \\
\vdots \\
y\left(k-n_{\mathrm{c}}\right)
\end{array}\right] \in \mathbb{R}^{n_{\mathrm{c}}\left(l_{u}+l_{y}\right)}
$$

where $y(k)=z(k)$, for all $k$.

\section{IV.B. Recursive Least Squares Update of $\theta(k)$}

We define the cumulative cost function

$$
J_{\mathrm{R}}(\theta(k)) \triangleq \sum_{i=q_{g}+1}^{k} \lambda^{k-i}\left\|\phi^{\mathrm{T}}\left(i-q_{g}-1\right) \theta^{\mathrm{T}}(k)-\hat{u}^{\mathrm{T}}\left(i-q_{g}\right)\right\|^{2}+\lambda^{k}(\theta(k)-\theta(0)) P^{-1}(0)(\theta(k)-\theta(0))^{\mathrm{T}},
$$

where $\|\cdot\|$ is the Euclidean norm and, for some $\varepsilon \in(0,1), \lambda(k) \in(\varepsilon, 1]$ is the forgetting factor and $\hat{u}(k)$ are the retrospectively optimized controls discussed in Section IV.C. Minimizing (20) yields

$$
\begin{aligned}
\theta^{\mathrm{T}}(k) \triangleq & \theta^{\mathrm{T}}(k-1)+\beta(k) P(k-1) \phi\left(k-q_{g}-1\right)\left[\phi^{\mathrm{T}}\left(k-q_{g}-1\right) P(k-1) \phi\left(k-q_{g}-1\right)+\lambda(k)\right]^{-1} \\
& \cdot\left[\theta(k-1) \phi\left(k-q_{g}-1\right)-\hat{u}\left(k-q_{g}\right)\right]^{\mathrm{T}},
\end{aligned}
$$


where $\beta(k)$ is either 0 or 1 . When $\beta(k)=1$, the controller is allowed to adapt, whereas, when $\beta(k)=0$, the adaptation is off. The error covariance is updated by

$$
\begin{aligned}
P(k) \triangleq & (1-\beta(k)) P(k-1)+\beta(k) \lambda^{-1}(k) P(k-1)-\beta(k) \lambda^{-1}(k) P(k-1) \phi\left(k-q_{g}-1\right) \\
\cdot & {\left[\phi^{\mathrm{T}}\left(k-q_{g}-1\right) P(k-1) \phi\left(k-q_{g}-1\right)+\lambda(k)\right]^{-1} \phi^{\mathrm{T}}\left(k-q_{g}-1\right) P(k-1) . }
\end{aligned}
$$

We initialize the error covariance matrix as $P(0)=\gamma I$, where $\gamma>0$.

\section{IV.C. Retrospectively Optimized Controls}

We solve for $\hat{\tilde{U}}(k)$, which has $\hat{u}\left(k-q_{g}\right)$ as a component, by minimizing the retrospective cost function

$$
\begin{aligned}
J(\hat{\tilde{U}}(k-1), k) & \triangleq \hat{Z}^{\mathrm{T}}(k) R_{1}(k) \hat{Z}(k)+\eta(k) \hat{\tilde{U}}^{\mathrm{T}}(k-1) R_{2}(k) \hat{\tilde{U}}(k-1) \\
& +\mu(k)[\hat{\tilde{U}}(k-1)+\hat{\tilde{U}}(k-2)]^{\mathrm{T}}[\hat{\tilde{U}}(k-1)+\hat{\tilde{U}}(k-2)],
\end{aligned}
$$

where $R_{1}(k) \in \mathbb{R}^{l_{z} s \times l_{z} s}$ is a positive-definite performance weighting, $R_{2}(k) \in \mathbb{R}^{g l_{u} \times g l_{u}}$ is a positive-definite control weighting, $\eta(k) \geq 0$ is a regularization weighting, and $\mu(k) \geq 0$ is a control rate penalty. The goal is to determine retrospective controls $\hat{\tilde{U}}(k-1)$ that would have provided better performance than the controls $U(k)$ that were applied to the system. The retrospectively optimized control values $\hat{\tilde{U}}(k-1)$ are then used to update the controller. Note that $\hat{Z}(k), Z(k), \tilde{\mathcal{H}}, \tilde{U}(k-1)$, and $\hat{\tilde{U}}(k-1)$ are developed in Appendix A.

Furthermore, $Z(k)$ is formed by stacking past performance variables $z(k)$, which for the 2D missile problem is defined as

$$
z(k) \triangleq M\left[A_{z, \mathrm{cmd}}-A_{z, \mathrm{IMU}}\right]
$$

Next, substituting (48) into (49) yields

$$
J(\hat{\tilde{U}}(k-1), k)=\hat{\tilde{U}}(k-1)^{\mathrm{T}} \mathcal{A}(k) \hat{\tilde{U}}(k-1)+\mathcal{B}^{\mathrm{T}}(k) \hat{\tilde{U}}^{\mathrm{T}}(k-1)+\mathcal{C}(k),
$$

where

$$
\begin{aligned}
\mathcal{A}(k) & \triangleq \tilde{\mathcal{H}}^{\mathrm{T}} R_{1}(k) \tilde{\mathcal{H}}+\eta(k) R_{2}(k)+\mu I, \\
\mathcal{B}(k) & \triangleq 2 \tilde{\mathcal{H}}^{\mathrm{T}} R_{1}(k)[Z(k)-\tilde{\mathcal{H}} \tilde{U}(k-1)]+2 \mu \hat{\tilde{U}}(k-2), \\
\mathcal{C}(k) & \triangleq Z^{\mathrm{T}}(k) R_{1}(k) Z(k)-2 Z^{\mathrm{T}}(k) R_{1}(k) \tilde{\mathcal{H}} \tilde{U}(k-1)+\tilde{U}^{\mathrm{T}}(k-1) \tilde{\mathcal{H}}^{\mathrm{T}} R_{1}(k) \tilde{\mathcal{H}} \tilde{U}(k-1) \\
& +\hat{\tilde{U}}^{\mathrm{T}}(k-2) \hat{\tilde{U}}(k-2) .
\end{aligned}
$$

If either $\tilde{\mathcal{H}}$ has full column rank or $\eta(k)>0$, then $\mathcal{A}(k)$ is positive definite. In this case, $J(\hat{\tilde{U}}(k-1), k)$ has the unique global minimizer

$$
\hat{\tilde{U}}(k-1)=-\frac{1}{2} \mathcal{A}^{-1}(k) \mathcal{B}(k),
$$

which is the optimized retrospective control. 


\section{Case 1 - Nominal Conditions}

For each scenario, a Monte Carlo simulation of 50 samples is run. The initial conditions of the target are varied for each of the runs. Table 4 gives the mean, distribution function, and standard deviation for each of the initial condition variables.

For all scenarios, the retrospective cost adaptive controller parameters are chosen as $n_{c}=5, \mathcal{H}=$ $\hat{H}_{1}=1, \theta(0)=0, \eta=0.01, \mu=500, \gamma=1 \times 10^{-5}, R_{1}=1$, and $R_{2}=1$. Note that $\hat{H}_{1}$ is an estimate of the first nonzero Markov parameter $H_{1}$ of the 2D missile. Although $H_{1}$ is a function of the trim condition, we choose $\hat{H}_{1}=1$, for all trim conditions. Furthermore, the adaptive controller is initialized at zero for every run of the Monte Carlo simulation. No baseline controller is used in conjunction with the adaptive controller.

\begin{tabular}{|c|c|c|c|}
\hline Variable & Mean & Distribution & Standard Deviation \\
\hline X - position & $4500[\mathrm{~m}]$ & Normal & $500[\mathrm{~m}]$ \\
Z - position & $3248[\mathrm{~m}]$ & Normal & $100[\mathrm{~m}]$ \\
Magnitude of target velocity & $328[\mathrm{~m} / \mathrm{s}]$ & Normal & $50[\mathrm{~m} / \mathrm{s}]$ \\
Body angle of target & $\pi[\mathrm{rad}]$ & Normal & $0.1[\mathrm{rad}]$ \\
\hline
\end{tabular}

Table 1. For each scenario the Monte Carlo simulation uses the initial conditions and distributions given in this table.

For the nominal scenario the Monte Carlo simulation yields a median miss distance for the autopilot of 0.12 meters, and for the adaptive control the median miss distance is 0.19 meters.

\begin{tabular}{|l|c|c|}
\hline Scenario & Autopilot Median Miss [m] & Adaptive Control Median Miss [m] \\
\hline Nominal & 0.12 & 0.19 \\
\hline
\end{tabular}

Table 2. Nominal median miss distance for the autopilot and adaptively controlled missile.

\section{V.A. Mitigation of Oscillatory Trajectories for Nominal Aerodynamics}

For the nominal scenario we examine a single run of the Monte Carlo simulation to evaluate the presence of oscillatory trajectories, which are undesirable. Figure 1 shows the trajectory of the missile and target for a single run. The normal acceleration and fin control are smooth, and the resulting miss distance is less than 1 meter.

Next, for this single example, apply RCAC with $\mu(k)=0$ for all $k \geq 0$. Figure 2 shows the trajectory of the missile and target for a single run. In this case, the normal acceleration and fin control contain high frequency content, which causes the missile to oscillate about its center of gravity resulting in a miss distance greater than 10 meters. Figure 3 shows the adaptive controller gains $\theta(k)$. Note the sharp jump in the control gains at approximately 0.8 seconds, approximately the time at which the seeker locks onto the target.

To eliminate the high frequency content in the missile trajectory, we now choose $\mu(k)=500$. Figure 4 shows the trajectory of the missile and target for same run as Figure 2. Note that the normal acceleration and fin control no longer contain high frequency content, this results in a smoother flight path, and a miss distance of less than 1 meter. Furthermore, as shown in Figure 5, the oscillation in the control gains is greatly reduced. 

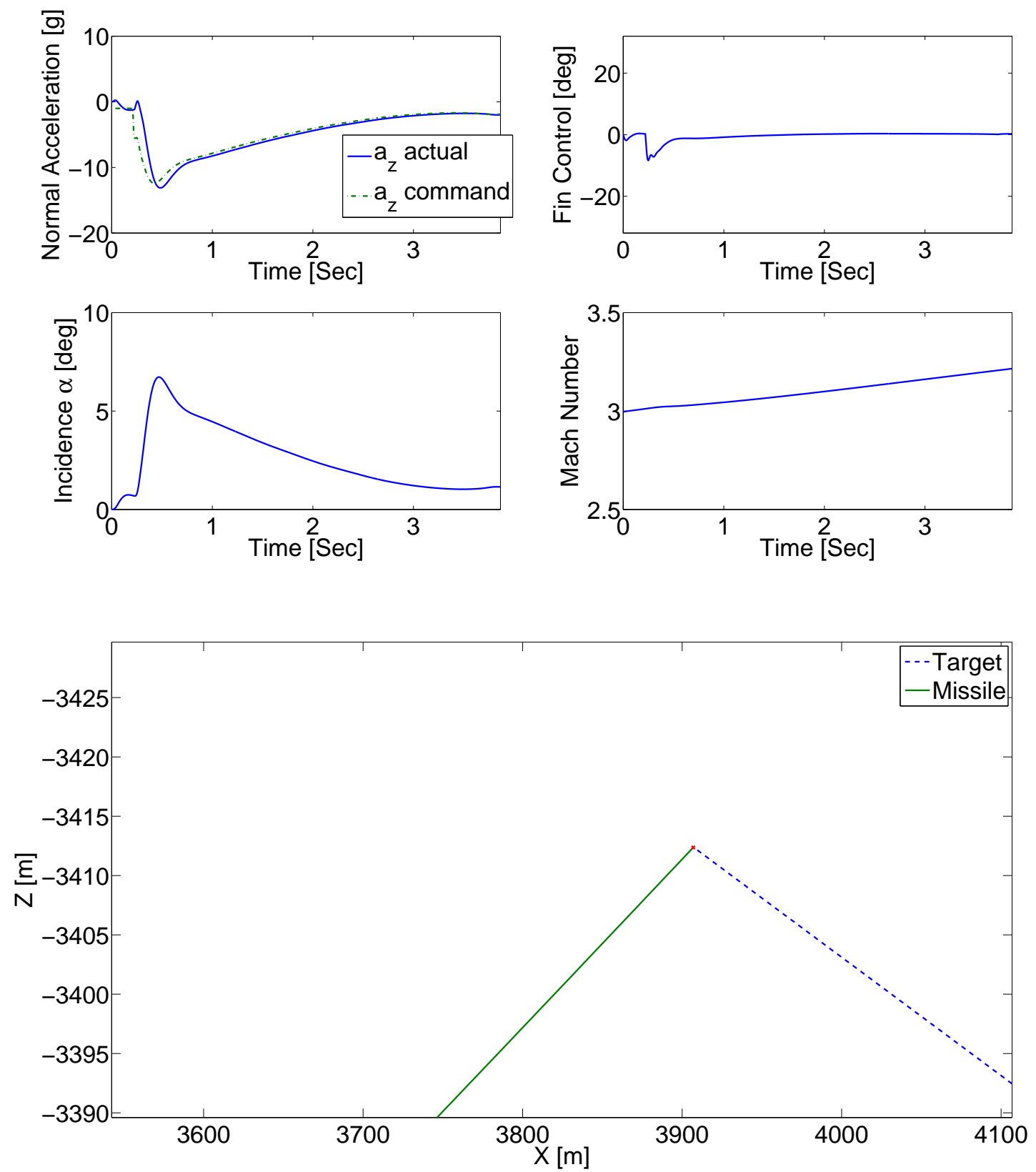

Figure 1. Autopilot controlled missile with nominal aerodynamic coefficients.

\section{Case 2 - Aerodynamic Force Coefficient}

In this set of scenarios we compare the median miss distance of the autopilot and the adaptively controlled missile when the aerodynamic force coefficient $C_{z}\left(\alpha, M, \delta_{p}\right)$, is modified by an affine linear function. Table 4 lists the scenarios and affine functions used to modify $C_{z}\left(\alpha, M, \delta_{p}\right)$. For this set of scenarios, the autopilot has a median miss distance ranging from 0.1 meters to 11.29 meters. The adaptively controlled missile has a median distance ranging from 0.17 meters to 35.77 meters. Note that the adaptive controller parameters and gains are not scheduled as in the autopilot setup. 

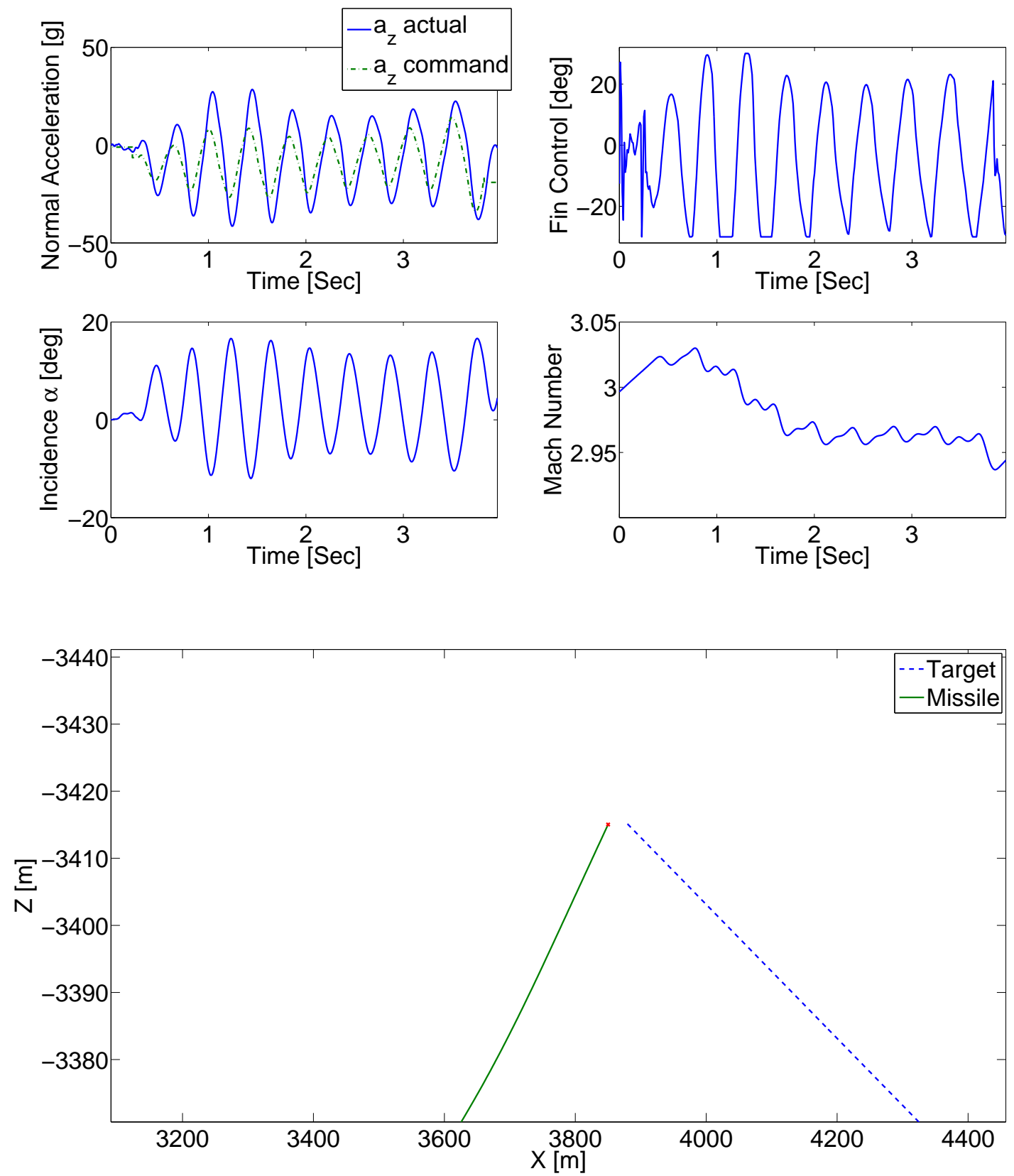

Figure 2. RCAC controlled missile with nominal aerodynamic coefficients. In this scenario, the oscillation penalty is removed from the adaptive control cost function.

\section{VI.A. Mitigation of Oscillatory Trajectories for Off-Nominal Aerodynamics}

For Scenario 3, we examine a single run of the Monte Carlo simulation to evaluate the presence of oscillatory trajectories. Figure 6 shows the autopilot controlled missile trajectory and the target for a single run. Note that in this scenario the normal acceleration and fin control oscillate at high frequency. This behavior creates a miss distance of 8.9 meters.

Choosing $\mu=500$, the trajectory of the adaptively controlled missile is smoothed out over the portion of the flight before the seeker locks onto the target, as shown in Figure 7. Furthermore, the adaptive control gains shown in Figure 8 converge to constant values. The resulting miss distance is 1.1 meters. 


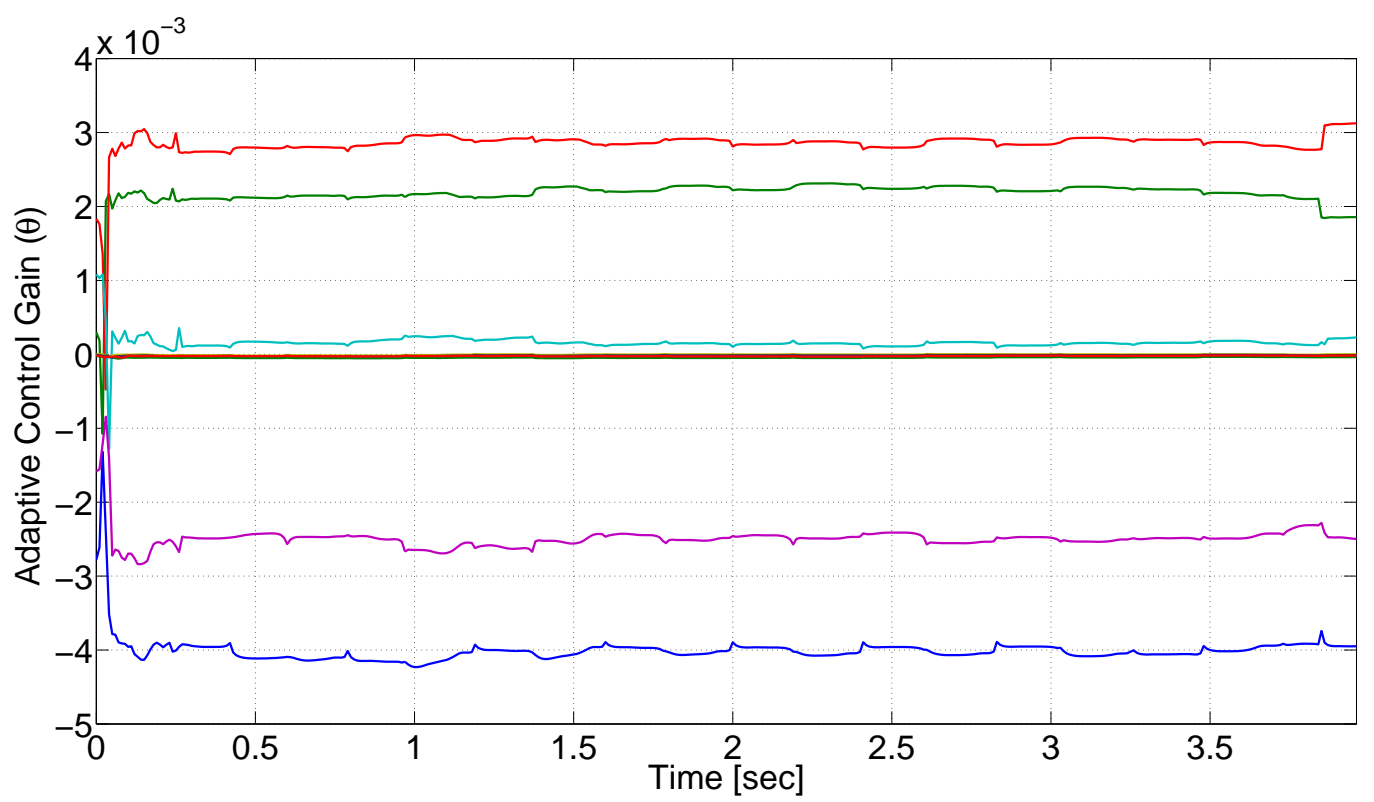

Figure 3. Adaptive control gains $\theta(k)$, where the missile has nominal aerodynamic coefficients. In this simulation, the RCAC cost function does not include an oscillation penalty term.

\begin{tabular}{|c|c|c|c|}
\hline Scenario & Autopilot Median Miss [m] & Adaptive Control Median Miss [m] & Aerodynamic Transformation \\
\hline 1 & 0.1 & 0.17 & $3 C_{z_{\alpha}}$ \\
2 & 2.74 & 0.89 & $3 C_{z_{\alpha}}+3$ \\
3 & 8.9 & 1.1 & $5 C_{z_{\alpha}}+5$ \\
4 & 0.35 & 4.67 & $0.75 C_{z_{\alpha}}$ \\
5 & 0.65 & 5.2 & $0.75 C_{z_{\alpha}}-0.75$ \\
6 & 11.29 & 35.77 & $0.5 C_{z_{\alpha}}-1$ \\
\hline
\end{tabular}

Table 3. Scenario and results for off-nominal aerodynamic force coefficients, specifically, the force coefficient $C_{z}\left(\alpha, M, \delta_{p}\right)$.

\section{Case 3 - Off-Nominal Aerodynamic Moment Coefficient}

In this set of scenarios we compare the median miss distance of the autopilot and the adaptively controlled missile when the aerodynamic moment coefficient $C_{z}\left(\alpha, M, \delta_{p}\right)$ is modified by an affine linear function. Table 4 lists the scenarios and affine functions used to modify $C_{m}\left(\alpha, M, \delta_{p}\right)$. For this set of scenarios, we demonstrate that both the autopilot and adaptive controller are robust to changes in the aerodynamic moment coefficient, the median miss distances are all under 1 meter.

\section{Case 4 - Noisy Pitch-Rate Sensor}

We now evaluate the autopilot and adaptive controller performance when noise is added to the pitch rate measurement $q$. Note that the autopilot uses the pitch rate directly for control. However, RCAC uses only the acceleration command-following error for feedback, although the seeker uses the pitch rate to generate the acceleration. Therefore, the adaptive controller is corrupted indirectly by sensor noise. Figure 9 shows the median miss distance in meters, for the both the autopilot and the adaptive controller for varying signal to noise ratios. Note that the performance of both controllers degrades as the noise increases, however, the adaptive controller degrades more slowly than the autopilot. The adaptive controller yields a smaller median miss distance at a signal to noise ratio of 400 . 

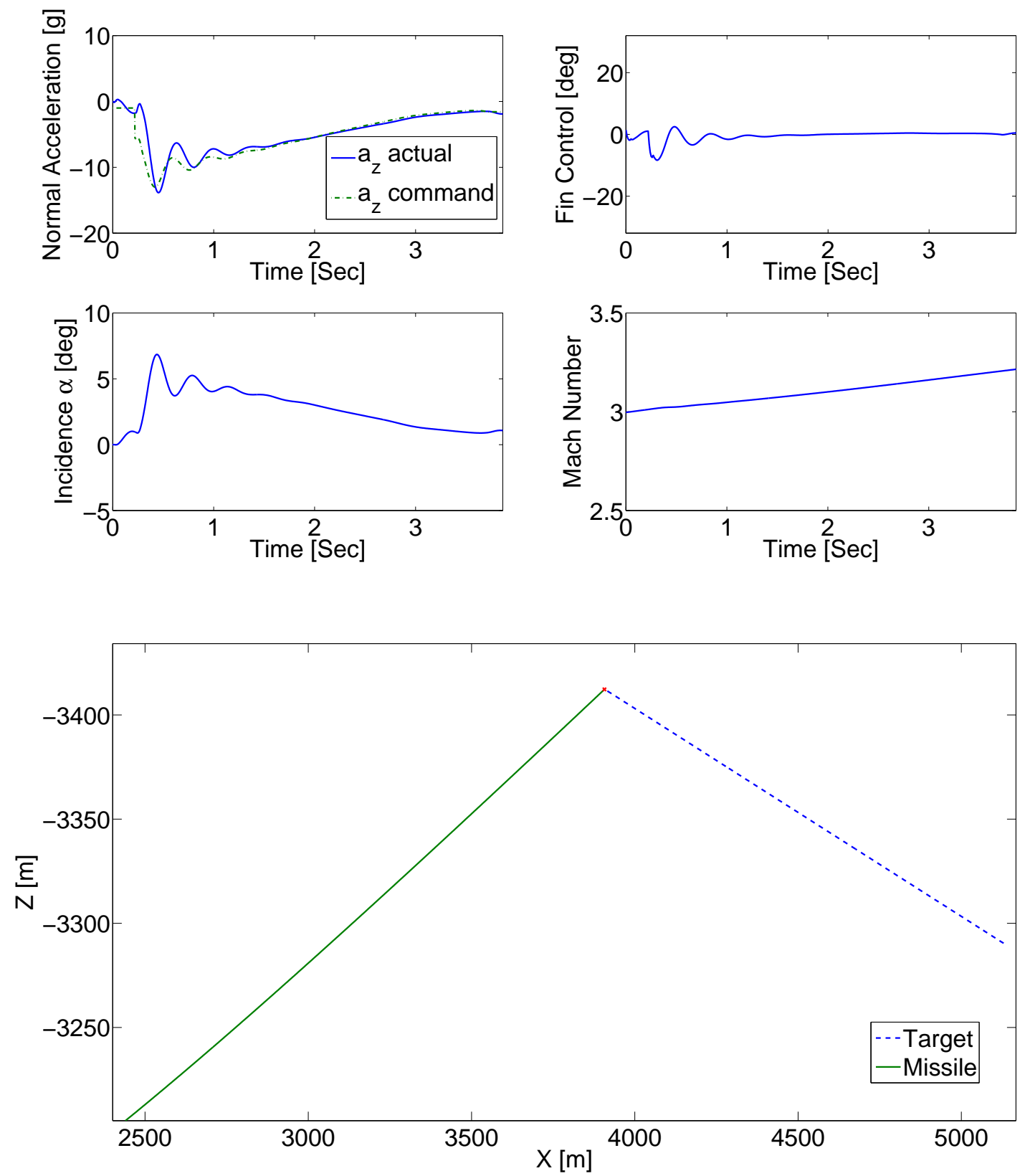

Figure 4. RCAC controlled missile with nominal aerodynamic coefficients. Furthermore, a penalty is added to the adaptive control cost function to penalize oscillatory trajectories.

\section{Conclusions}

In this paper we extended the results presented in [3] by using recent developments in retrospective cost adaptive control $[7,8]$. In previous work, the adaptive controller was used in an outer loop around the autopilot, since RCAC alone failed to stabilize the missile for most tactical trajectories. The extensions in RCAC, which eliminate the need for knowledge of nonminimum-phase zero locations, allow us to eliminate the autopilot in the the inner loop. We demonstrated that the adaptive controller yields performance that is comparable to the gain scheduled three-loop autopilot for the nominal case. Furthermore, we demonstrated that in certain off-nominal scenarios, the adaptive controller outperforms the three-loop autopilot. In all cases, the tuning of both the autopilot and adaptive controller is fixed, that is, the tuning parameters are 


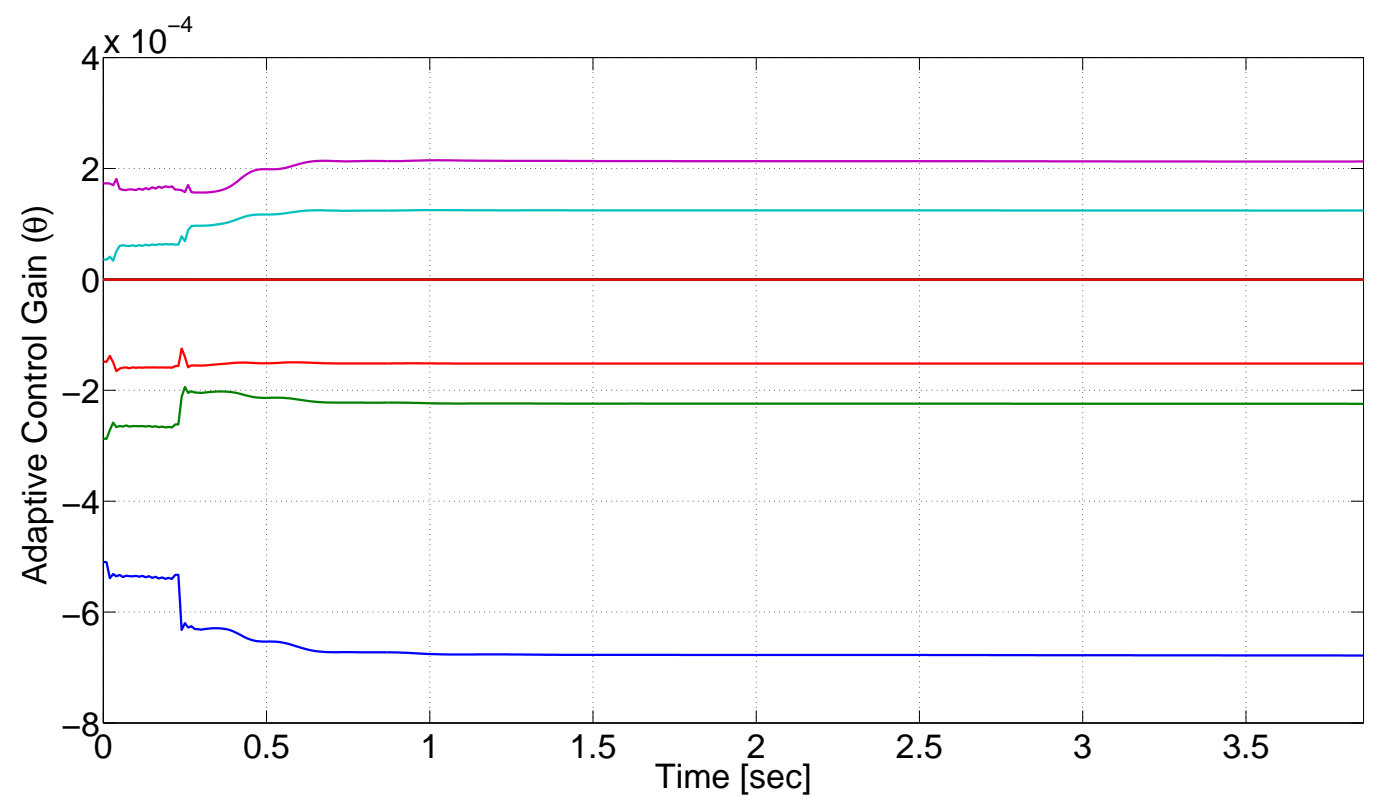

Figure 5. Adaptive control gains $\theta(k)$, where the missile has nominal aerodynamic coefficients. Furthermore, a penalty is added to the adaptive control cost function to penalize oscillatory trajectories.

\begin{tabular}{|c|c|c|c|}
\hline Scenario & Autopilot Miss $[\mathrm{m}]$ & Adaptive Control Miss $[\mathrm{m}]$ & Aerodynamic Transformation \\
\hline 1 & 0.08 & 0.31 & $0.5 C_{m_{\alpha}}$ \\
2 & 0.08 & 0.61 & $0.5 C_{m_{\alpha}}-0.5$ \\
3 & 0.07 & 0.24 & $0.5 C_{m_{\alpha}}+0.5$ \\
4 & 0.09 & 0.2 & $0.25 C_{m_{\alpha}}$ \\
5 & 0.07 & 0.44 & $0.25 C_{m_{\alpha}}+1$ \\
6 & 0.06 & 0.16 & $0.25 C_{m_{\alpha}}-1$ \\
\hline
\end{tabular}

Table 4. Scenario and results for the off-nominal aerodynamic coefficients, specifically, in the moment coefficient $C_{m}\left(\alpha, M, \delta_{p}, q\right)$

not modified for each scenario.

\section{Appendix}

\section{A. RCAC Algorithm}

Consider the MIMO discrete-time system

$$
\begin{aligned}
x(k+1) & =A x(k)+B u(k)+D_{1} w(k), \\
y(k) & =C x(k)+D_{2} w(k), \\
z(k) & =E_{1} x(k)+E_{0} w(k),
\end{aligned}
$$

where $x(k) \in \mathbb{R}^{n}, y(k) \in \mathbb{R}^{l_{y}}, z(k) \in \mathbb{R}^{l_{z}}, u(k) \in \mathbb{R}^{l_{u}}, w(k) \in \mathbb{R}^{l_{w}}, k \geq 0,(A, B)$ is controllable, and $(A, C)$ and $(A, E)$ are observable. Although the state $x(k)$ is used for analysis, the algorithm described in this paper does not rely on a state space representation. The goal is to develop an adaptive output feedback controller that minimizes the performance variable $z$ in the presence of the exogenous signal $w$ with minimal modeling information about the dynamics and $w$. 

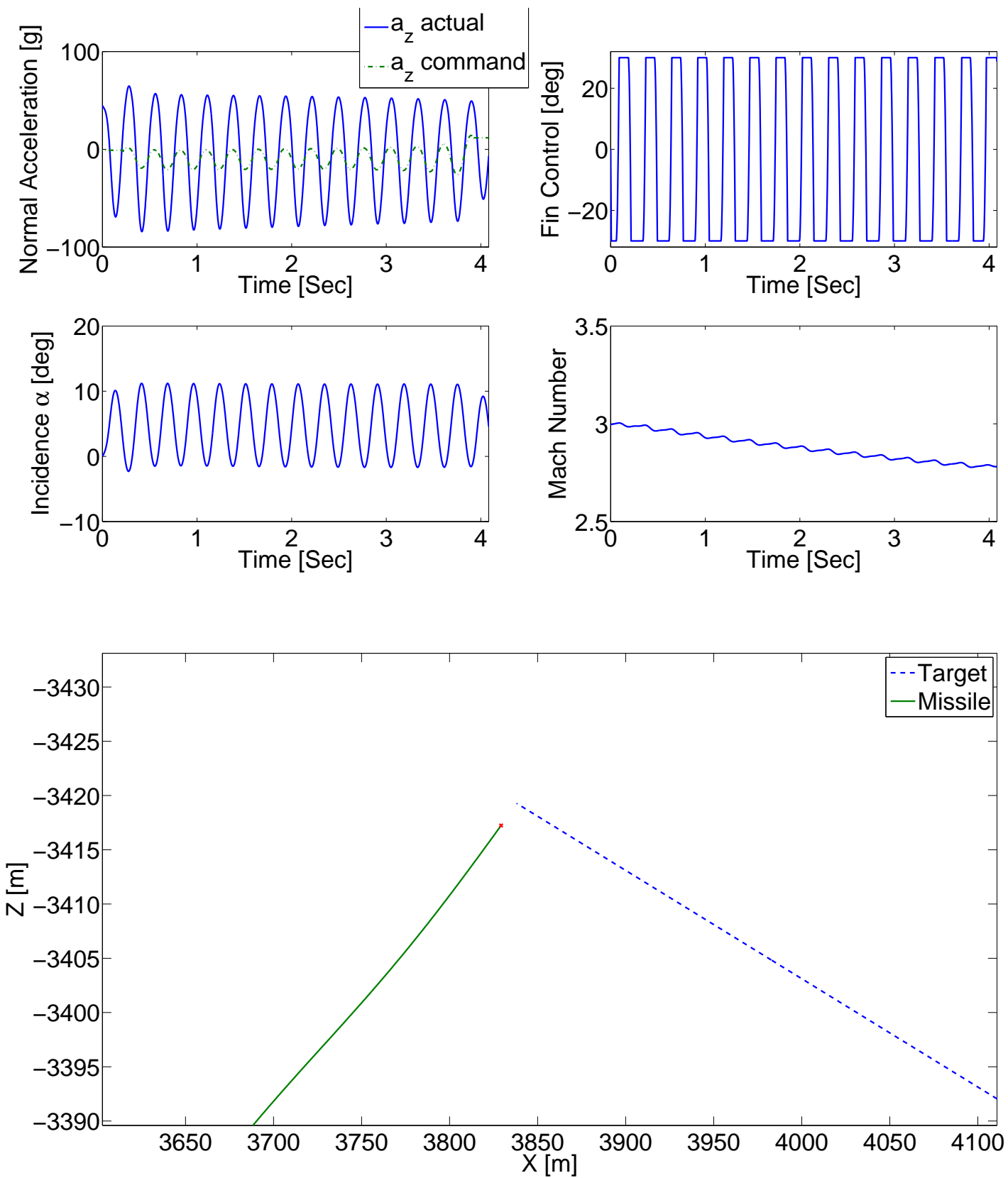

Figure 6. Autopilot controlled missile with off-nominal aerodynamic coefficients. In this case $C_{z}\left(\alpha, M, \delta_{p}\right)$ is perturbed by an affine linear transformation.

Note that $w$ can represent either a command signal to be followed, an external disturbance to be rejected, or both. In particular if $D_{1}=0$ and $E_{0} \neq 0$, then the objective is to have the output $E_{1} x$ follow the command signal $-E_{0} w$. On the other hand, if $D_{1} \neq 0$ and $E_{0}=0$, then the objective is to reject the disturbance $w$ from the performance measurement $E_{1} x$. Furthermore, if $D_{1}=\left[\begin{array}{ll}\hat{D}_{1} & 0\end{array}\right]$, $E_{0}=\left[\begin{array}{cc}0 & \hat{E}_{0}\end{array}\right]$, and $w(k)=\left[w_{1}(k)^{\mathrm{T}} w_{2}(k)^{\mathrm{T}}\right]^{\mathrm{T}}$, then the objective is to have $E_{1} x$ follow the command $-\hat{E}_{0} w_{2}$ while rejecting the disturbance $w_{1}$. Finally, if $D_{1}$ and $E_{0}$ are empty matrices, then the objective is output stabilization, that is, convergence of $z$ to zero. 

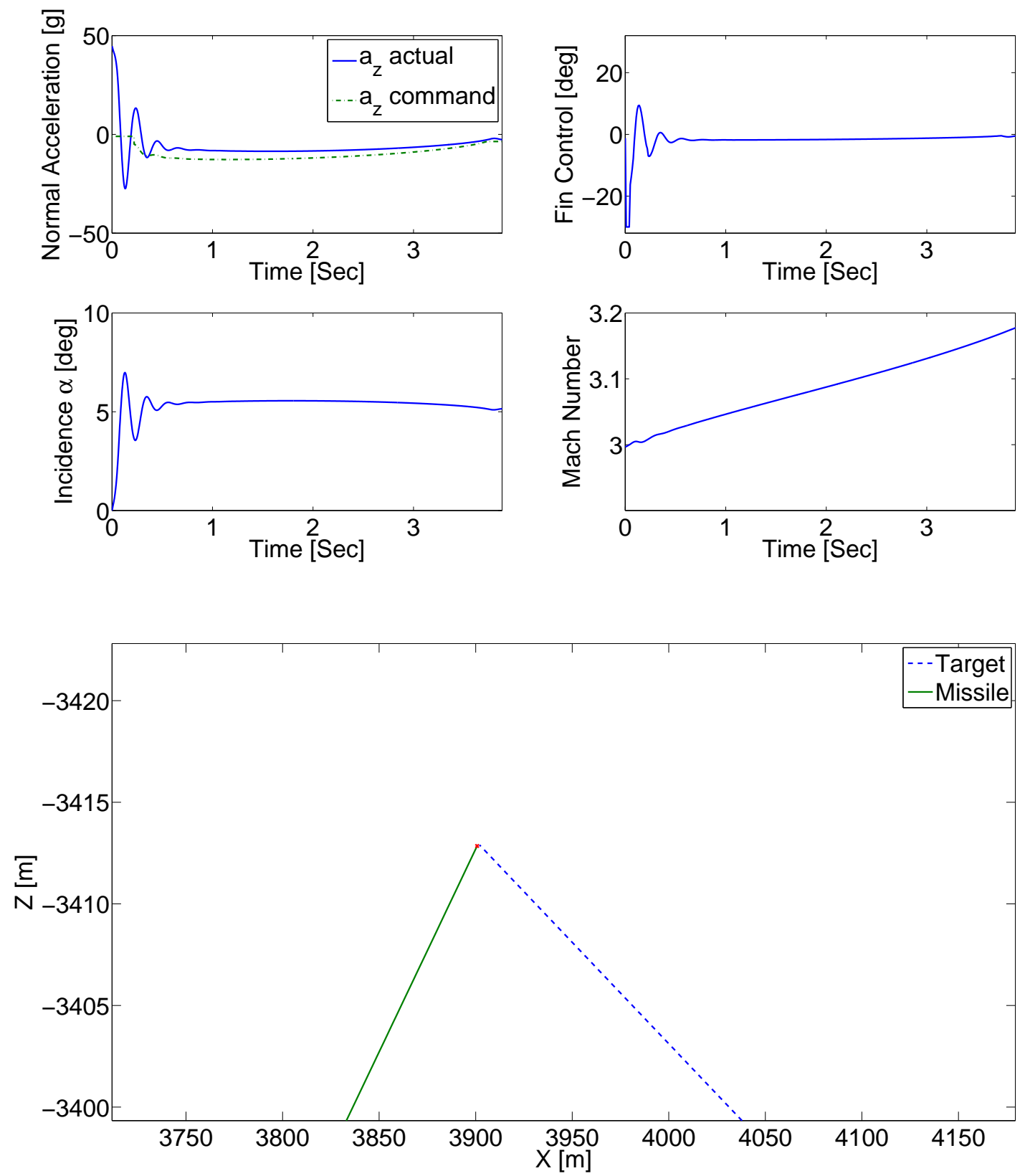

Figure 7. RCAC controlled missile with off-nominal aerodynamic coefficients. In this case $C_{z}\left(\alpha, M, \delta_{p}\right)$ is perturbed by an affine linear transformation. Furthermore, a penalty is added to the RCAC cost function to penalize oscillatory trajectories.

\section{A.A. Development of Retrospective Cost function}

For $i \geq 1$, define the Markov parameter of $G_{z u}$ given by

$$
H_{i} \triangleq E_{1} A^{i-1} B
$$

For example, $H_{1}=E_{1} B$ and $H_{2}=E_{1} A B$. Let $r$ be a positive integer. Then, for all $k \geq r$,

$$
x(k)=A^{r} x(k-r)+\sum_{i=1}^{r} A^{i-1} B u(k-i)+\sum_{i=1}^{r} A^{i-1} D_{1} w(k-i),
$$




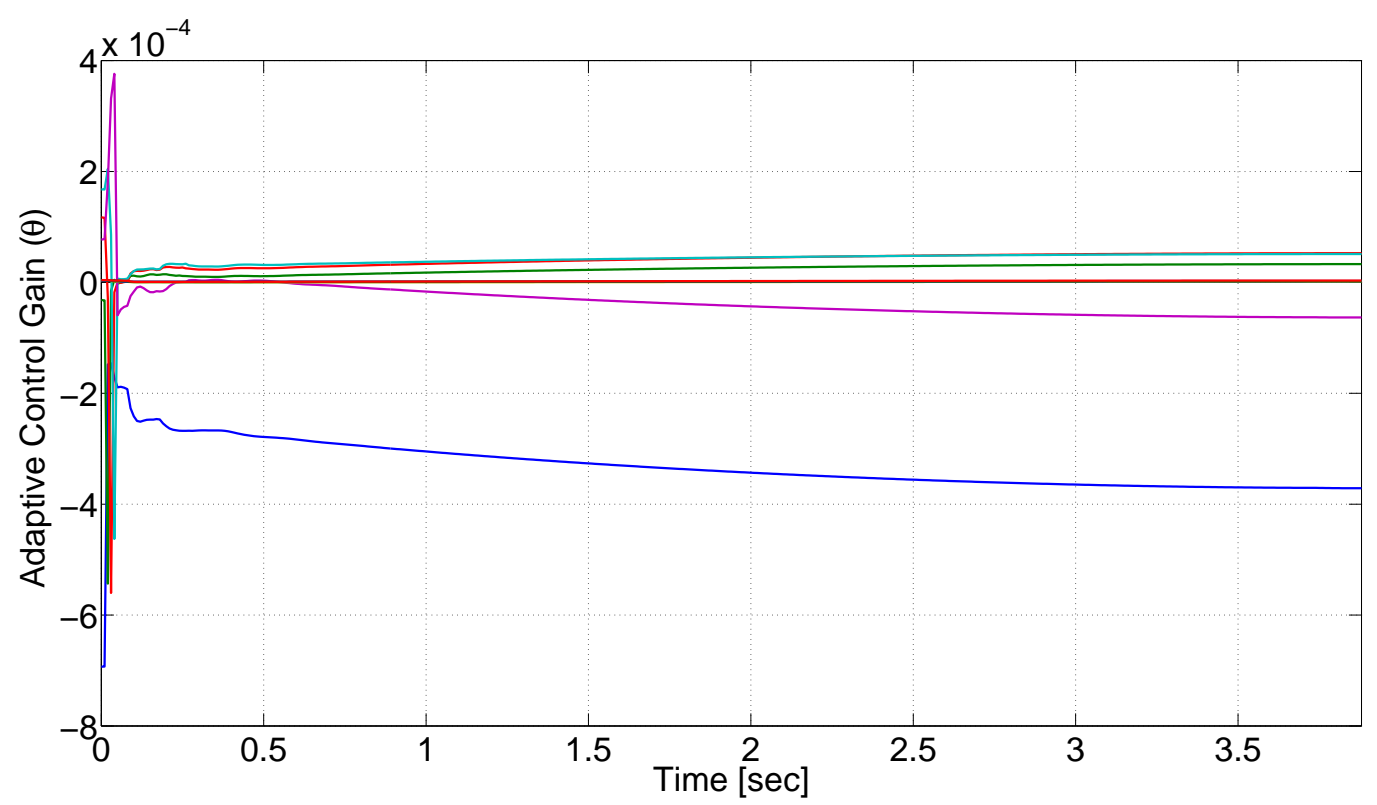

Figure 8. Adaptive control gains $\theta(k)$, where $C_{z}\left(\alpha, M, \delta_{p}\right)$ is perturbed by an affine linear transformation. In this scenario, the cost function includes an oscillation penalty.

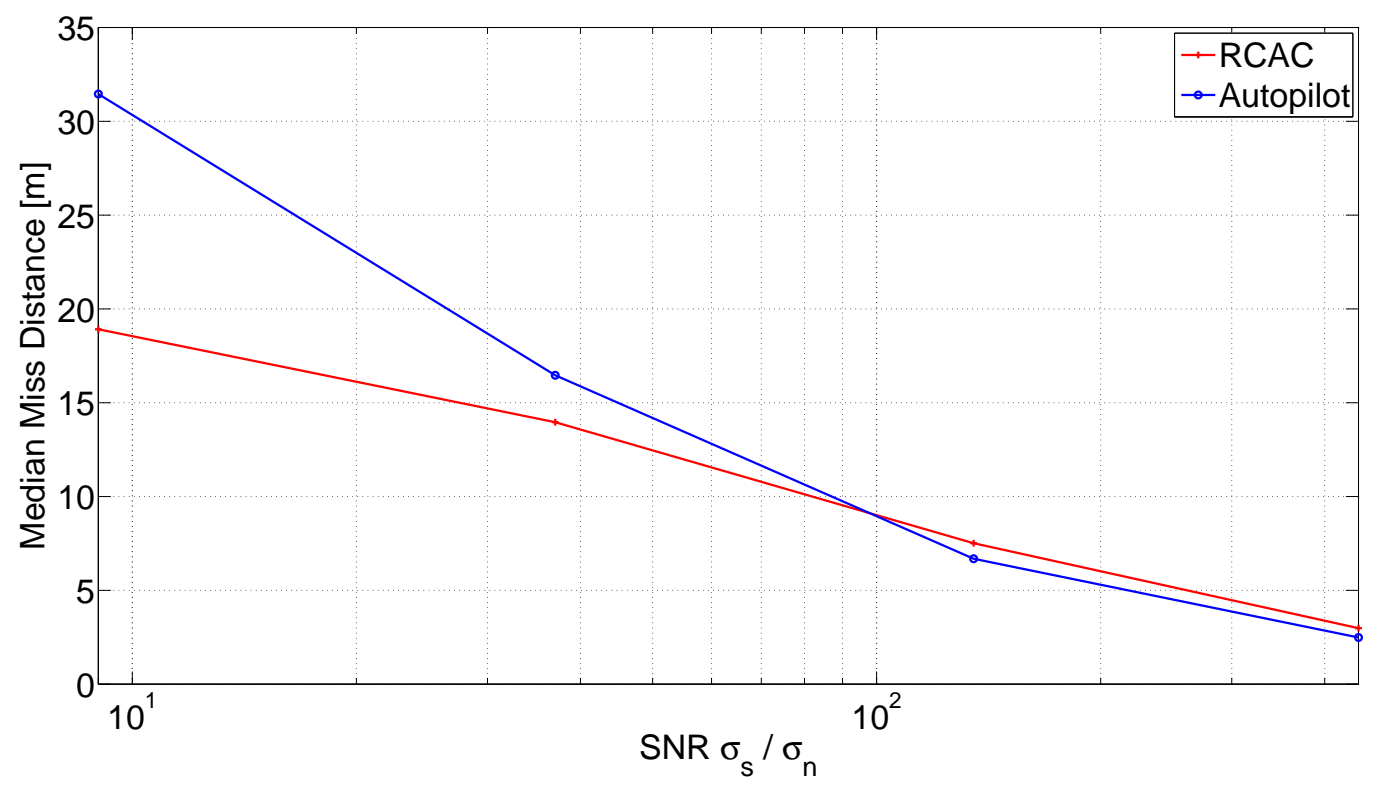

Figure 9. Comparison between RCAC controlled missile and autopilot controlled missile under nominal flight conditions. In this scenario we consider noise on the sensing of $q$. For each point, a Monte Carlo run of 50 is used to determine the median miss distance.

and thus

$$
z(k)=E_{1} A^{r} x(k-r)+\sum_{i=1}^{r} E_{1} A^{i-1} D_{1} w(k-i)+E_{0} w(k)+\bar{H} \bar{U}(k-1),
$$

where

$$
\bar{H} \triangleq\left[\begin{array}{lll}
H_{1} & \cdots & H_{r}
\end{array}\right] \in \mathbb{R}^{l_{z} \times r l_{u}}
$$

\section{4 of 18}


and

$$
\bar{U}(k-1) \triangleq\left[\begin{array}{c}
u(k-1) \\
\vdots \\
u(k-r)
\end{array}\right] .
$$

Next, we rearrange the columns of $\bar{H}$ and the components of $\bar{U}(k-1)$ and partition the resulting matrix and vector so that

$$
\bar{H} \bar{U}(k-1)=\mathcal{H}^{\prime} U^{\prime}(k-1)+\mathcal{H} U(k-1),
$$

where $\mathcal{H}^{\prime} \in \mathbb{R}^{l_{z} \times\left(r l_{u}-l_{U}\right)}, \mathcal{H} \in \mathbb{R}^{l_{z} \times l_{U}}, U^{\prime}(k-1) \in \mathbb{R}^{r l_{u}-l_{U}}$, and $U(k-1) \in \mathbb{R}^{l_{U}}$. Then, we can rewrite (35) as

$$
z(k)=\mathcal{S}(k)+\mathcal{H} U(k-1),
$$

where

$$
\mathcal{S}(k) \triangleq E_{1} A^{r} x(k-r)+\sum_{i=1}^{r} E_{1} A^{i-1} D_{1} w(k-i)+E_{0} w(k)+\mathcal{H}^{\prime} U^{\prime}(k-1) .
$$

For example, $\bar{H}=\left[\begin{array}{lllll}H_{1} & H_{2} & H_{3} & H_{4} & H_{5}\end{array}\right]$,

$$
\mathcal{H}^{\prime}=\left[\begin{array}{lll}
H_{1} & H_{2} & H_{4}
\end{array}\right], \quad U^{\prime}(k-1)=\left[\begin{array}{c}
u(k-1) \\
u(k-2) \\
u(k-4)
\end{array}\right],
$$

and

$$
\mathcal{H}=\left[\begin{array}{ll}
H_{3} & H_{5}
\end{array}\right], \quad U(k-1)=\left[\begin{array}{l}
u(k-3) \\
u(k-5)
\end{array}\right] .
$$

Next, let $s$ denote the number of delays, therefore for $j=1, \ldots, s$, we rewrite (37) with a delay of $k_{j}$ time steps, where $0 \leq k_{1} \leq k_{2} \leq \cdots \leq k_{s}$, in the form

$$
z\left(k-k_{j}\right)=\mathcal{S}_{j}\left(k-k_{j}\right)+\mathcal{H}_{j} U_{j}\left(k-k_{j}-1\right),
$$

where (38) becomes

$$
\mathcal{S}_{j}\left(k-k_{j}\right) \triangleq E_{1} A^{r} x\left(k-k_{j}-r\right)+\sum_{i=1}^{r} E_{1} A^{i-1} D_{1} w\left(k-k_{j}-i\right)+E_{0} w\left(k-k_{j}\right)+\mathcal{H}_{j}^{\prime} U_{j}^{\prime}\left(k-k_{j}-1\right)
$$

and (36) becomes

$$
\bar{H} \bar{U}\left(k-k_{j}-1\right)=\mathcal{H}_{j}^{\prime} U_{j}^{\prime}\left(k-k_{j}-1\right)+\mathcal{H}_{j} U_{j}\left(k-k_{j}-1\right),
$$

where $\mathcal{H}_{j}^{\prime} \in \mathbb{R}^{l_{z} \times\left(r l_{u}-l_{U_{j}}\right)}, \mathcal{H}_{j} \in \mathbb{R}^{l_{z} \times l_{U_{j}}}, U_{j}^{\prime}\left(k-k_{j}-1\right) \in \mathbb{R}^{r l_{u}-l_{U_{j}}}$, and $U_{j}\left(k-k_{j}-1\right) \in \mathbb{R}^{l_{U_{j}}}$. Now, by stacking $z\left(k-k_{1}\right), \ldots, z\left(k-k_{s}\right)$, we define the extended performance

$$
Z(k) \triangleq\left[\begin{array}{c}
z\left(k-k_{1}\right) \\
\vdots \\
z\left(k-k_{s}\right)
\end{array}\right] \in \mathbb{R}^{s l_{z}} .
$$

Therefore,

$$
Z(k) \triangleq \tilde{\mathcal{S}}(k)+\tilde{\mathcal{H}} \tilde{U}(k-1),
$$


where

$$
\tilde{\mathcal{S}}(k) \triangleq\left[\begin{array}{c}
\mathcal{S}_{1}\left(k-k_{1}\right) \\
\vdots \\
\mathcal{S}_{s}\left(k-k_{s}\right)
\end{array}\right] \in \mathbb{R}^{s l_{z}}
$$

$\tilde{U}(k-1)$ has the form

$$
\tilde{U}(k-1) \triangleq\left[\begin{array}{c}
u\left(k-q_{1}\right) \\
\vdots \\
u\left(k-q_{g}\right)
\end{array}\right] \in \mathbb{R}^{g l_{u}},
$$

where, for $i=1, \ldots, g, k_{1} \leq q_{i} \leq k_{s}+r$, and $\tilde{\mathcal{H}} \in \mathbb{R}^{s l_{z} \times g l_{u}}$ is constructed according to the structure of $\tilde{U}(k-1)$. The vector $\tilde{U}(k-1)$ is formed by stacking $U_{1}\left(k-k_{1}-1\right), \ldots, U_{s}\left(k-k_{s}-1\right)$ and removing copies of repeated components.

For example, with $k_{1}=0$ and $k_{2}=2$, stacking $U_{1}(k-1)=\left[\begin{array}{c}u(k-1) \\ u(k-2)\end{array}\right]$ and $U_{2}(k-3)=u(k-3)$ results in $\tilde{U}(k-1)=\left[\begin{array}{l}u(k-1) \\ u(k-2) \\ u(k-3)\end{array}\right]$ and $\tilde{\mathcal{H}}=\left[\begin{array}{ccc}H_{1} & H_{2} & H_{3} \\ 0 & H_{1} & H_{2} \\ 0 & 0 & H_{3}\end{array}\right]$. Note that $\tilde{\mathcal{H}}$ consists of the entries of $\mathcal{H}_{1}, \ldots, \mathcal{H}_{s}$ arranged according to the structure of $\tilde{U}(k-1)$.

Next, we define the retrospective performance

$$
\hat{z}\left(k-k_{j}\right) \triangleq \mathcal{S}_{j}\left(k-k_{j}\right)+\mathcal{H}_{j} \hat{U}_{j}\left(k-k_{j}-1\right),
$$

where the past controls $U_{j}\left(k-k_{j}-1\right)$ in (39) are replaced by the retrospective controls $\hat{U}_{j}\left(k-k_{j}-1\right)$. In analogy with (41), the extended retrospective performance is defined as

$$
\hat{Z}(k) \triangleq\left[\begin{array}{c}
\hat{z}\left(k-k_{1}\right) \\
\vdots \\
\hat{z}\left(k-k_{s}\right)
\end{array}\right] \in \mathbb{R}^{s l_{z}}
$$

and thus is given by

$$
\hat{Z}(k)=\tilde{\mathcal{S}}(k)+\tilde{\mathcal{H}} \hat{\tilde{U}}(k-1),
$$

where the components of $\hat{\tilde{U}}(k-1) \in \mathbb{R}^{l} \tilde{U}$ are the components of $\hat{U}_{1}\left(k-k_{1}-1\right), \ldots, \hat{U}_{s}\left(k-k_{s}-1\right)$ ordered in the same way as the components of $\tilde{U}(k-1)$. Subtracting (42) from (47) yields

$$
\hat{Z}(k)=Z(k)-\tilde{\mathcal{H}} \tilde{U}(k-1)+\tilde{\mathcal{H}} \hat{\tilde{U}}(k-1) .
$$

Finally, we define the retrospective cost function

$$
\begin{aligned}
J(\hat{\tilde{U}}(k-1), k) & \triangleq \hat{Z}^{\mathrm{T}}(k) R_{1}(k) \hat{Z}(k)+\eta(k) \hat{\tilde{U}}^{\mathrm{T}}(k-1) R_{2}(k) \hat{\tilde{U}}(k-1) \\
& +\mu(k)[\hat{\tilde{U}}(k-1)+\hat{\tilde{U}}(k-2)]^{\mathrm{T}}[\hat{\tilde{U}}(k-1)+\hat{\tilde{U}}(k-2)],
\end{aligned}
$$

where $R_{1}(k) \in \mathbb{R}^{l_{z} s \times l_{z} s}$ is a positive-definite performance weighting, $R_{2}(k) \in \mathbb{R}^{g l_{u} \times g l_{u}}$ is a positive-definite control weighting, $\eta(k) \geq 0$ is a regularization weighting, and $\mu(k) \geq 0$ is a control rate penalty. The goal is 
to determine retrospective controls $\hat{\tilde{U}}(k-1)$ that would have provided better performance than the controls $U(k)$ that were applied to the system. The retrospectively optimized control values $\hat{\tilde{U}}(k-1)$ are then used to update the controller.

Next, substituting (48) into (49) yields

$$
J(\hat{\tilde{U}}(k-1), k)=\hat{\tilde{U}}(k-1)^{\mathrm{T}} \mathcal{A}(k) \hat{\tilde{U}}(k-1)+\mathcal{B}^{\mathrm{T}}(k) \hat{\tilde{U}}^{\mathrm{T}}(k-1)+\mathcal{C}(k),
$$

where

$$
\begin{aligned}
\mathcal{A}(k) & \triangleq \tilde{\mathcal{H}}^{\mathrm{T}} R_{1}(k) \tilde{\mathcal{H}}+\eta(k) R_{2}(k)+\mu I, \\
\mathcal{B}(k) & \triangleq 2 \tilde{\mathcal{H}}^{\mathrm{T}} R_{1}(k)[Z(k)-\tilde{\mathcal{H}} \tilde{U}(k-1)]+2 \mu \hat{\tilde{U}}(k-2), \\
\mathcal{C}(k) & \triangleq Z^{\mathrm{T}}(k) R_{1}(k) Z(k)-2 Z^{\mathrm{T}}(k) R_{1}(k) \tilde{\mathcal{H}} \tilde{U}(k-1)+\tilde{U}^{\mathrm{T}}(k-1) \tilde{\mathcal{H}}^{\mathrm{T}} R_{1}(k) \tilde{\mathcal{H}} \tilde{U}(k-1) \\
& +\hat{\tilde{U}}^{\mathrm{T}}(k-2) \hat{\tilde{U}}(k-2) .
\end{aligned}
$$

If either $\tilde{\mathcal{H}}$ has full column rank or $\eta(k)>0$, then $\mathcal{A}(k)$ is positive definite. In this case, $J(\hat{\tilde{U}}(k-1), k)$ has the unique global minimizer

$$
\hat{\tilde{U}}(k-1)=-\frac{1}{2} \mathcal{A}^{-1}(k) \mathcal{B}(k),
$$

which is the optimized retrospective control.

The regularization weighting $\eta(k)$ can be used to bound control effort. For example, $\eta(k)$ may be performance based

$$
\eta(k)=\eta_{0}(k)\|Z(k)\|_{2}^{2}
$$

or control-error based

$$
\eta(k)=\eta_{0}(k)|| \hat{\tilde{U}}(k-2)-\tilde{U}(k-2) \|_{2}^{2},
$$

where $\eta_{0}(k) \geq 0$. Alternatively, the control effort can be bounded using a saturation function where $\eta(k) \equiv 0$ and (54) is replaced by

$$
\hat{\tilde{U}}(k-1) \triangleq \operatorname{sat}_{[a, b]}\left[-\frac{1}{2} \mathcal{A}^{-1}(k) \mathcal{B}(k)\right]
$$

where $\operatorname{sat}_{[a, b]}(\zeta)$ is the component-wise saturation function that, for scalar arguments, is defined by

$$
\operatorname{sat}_{[a, b]}(\zeta) \triangleq \begin{cases}b, & \text { if } \zeta \geq b \\ \zeta, & \text { if } a<\zeta<b \\ a & \text { if } \zeta \leq a\end{cases}
$$

where $a<b$ are the component-wise saturation levels.

\section{References}

\footnotetext{
${ }^{1}$ Narendra, K. and Annaswamy, A., Stable Adaptive Systems, Prentice-Hall, 1989.

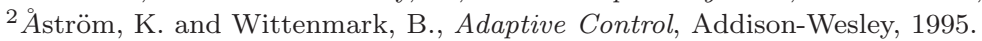

${ }^{3}$ R. J. Fuentes, J. B. Hoagg, B. J. Anderton, A. M. D'Amato, and D. S. Bernstein, "Investigation of Cumulative Retrospective Cost Adaptive Control for Missile Application," Proc. AIAA Guidance, Navigation and Control Conference, AIAA-2010-7577, Toronto, ON, August 2010.

${ }^{4}$ J. B. Hoagg and D. S. Bernstein, "Retrospective Cost Adaptive Control for Nonminimum-Phase Discrete-Time Systems Part 1: The Ideal Controller and Error System; Part 2: The Adaptive Controller and Stability Analysis," Proc. Conf. Dec. Contr., pp. 893-904, Atlanta, GA, December 2010.
} 
${ }^{5}$ J. B. Hoagg and D. S. Bernstein, "Retrospective Cost Model Reference Adaptive Control for Nonminimum-Phase DiscreteTime Systems, Part 1: The Adaptive Controller; Part 2: Stability Analysis," Proc. Amer. Contr. Conf., pp. 2927-2938, San Francisco, CA, June 2011.

${ }^{6}$ Mracek, C. and Ridgely, D., "Missile Longitudinal Autopilots: Connections between Optimal Control and Classical Topologies," Proc. AIAA Guid., Nav. Contr. Conf., AIAA 2005-6381, San Francisco, CA, 2005.

7 A. M. D'Amato and D. S. Bernstein, "Adaptive Forward-Propagation Input Reconstruction for Nonminimum-Phase Systems," Proc. Amer. Conf. Contr., Montreal, Canada, June 2012.

8 A. M. D'Amato, E. D. Sumer, and D. S. Bernstein, "Frequency-Domain Stability Analysis of Retrospective-Cost Adaptive Control for Systems with Unknown Nonminimum-Phase Zeros," Proc. Conf. Dec. Contr., Orlando, FL, December 2011. 